\title{
Distribution of emm type and antibiotic susceptibility of group A streptococci causing invasive and noninvasive disease
}

\begin{abstract}
Correspondence
Kimiko Ubukata

ubukatak@lisci.kitasato-u.ac.jp
\end{abstract}

Received 14 April 2008

Accepted 28 July 2008

\section{Takeaki Wajima, ${ }^{1}$ Somay Y. Murayama, ${ }^{1}$ Katsuhiko Sunaoshi, ${ }^{1}$ Eiichi Nakayama, ${ }^{1}$ Keisuke Sunakawa ${ }^{2}$ and Kimiko Ubukata ${ }^{1}$}

${ }^{1}$ Laboratory of Molecular Epidemiology for Infectious Agents, Graduate School of Infection Control Sciences, Kitasato University, Tokyo, Japan

${ }^{2}$ Laboratory of Infectious Disease, Graduate School of Infection Control Sciences, Kitasato University, Tokyo, Japan

To determine the prevalence of macrolide antibiotic and levofloxacin resistance in infections with Streptococcus pyogenes (group A streptococcus or GAS), strains were collected from 45 medical institutions in various parts of Japan between October 2003 and September 2006. Four hundred and eighty-two strains from patients with GAS infections were characterized genetically. Strains were classified into four groups according to the type of infection: invasive infections $(n=74)$ including sepsis, cellulitis and toxic-shock-like syndrome; acute otitis media (AOM; $n=23)$; abscess $(n=53)$; and pharyngotonsillitis $(n=332)$. Among all strains, $32 \mathrm{emm}$ types were identified; emm1 was significantly more common in invasive infections (39.2\%) and AOM $(43.5 \%)$ than in abscesses (3.8\%) or pharyngotonsillitis (10.2\%). emm12 and emm4 each accounted for $23.5 \%$ of pharyngotonsillitis cases. Susceptibility of GAS strains to eight $\beta$-lactam agents was excellent, with MICs of $0.0005-0.063 \mu^{-1} ~ m^{-1}$. Macrolide-resistant strains accounted for $16.2 \%$ of all strains, while the percentages of strains possessing the resistance genes erm $(A)$, $\operatorname{erm}(\mathrm{B})$ and $\operatorname{mef}(\mathrm{A})$ were $2.5 \%, 6.2 \%$ and $7.5 \%$, respectively. Although no strains with high resistance to levofloxacin were found, strains with an $\mathrm{MIC}$ of $2-4 \mu \mathrm{g} \mathrm{ml}^{-1}$ (17.4\%) had amino acid substitutions at either Ser-79 or Asp-83 in ParC. These levofloxacin-intermediately resistant strains included $16 \mathrm{emm}$ types, but macrolide-resistant strains were more likely than others to represent certain emm types.

\section{INTRODUCTION}

Streptococcus pyogenes (group A streptococcus or GAS) is well recognized as the most common pathogen causing pharyngotonsillitis in school-age children. In addition, GAS is well known as a cause of impetigo, necrotizing fasciitis and other infections (Cunningham, 2000). In the late 1980s, toxic-shock-like syndrome caused by GAS was reported in Europe, Japan and the USA, being termed 'flesh-eating bacteria' in the popular press (Cunningham, 2000; Stevens et al., 1989).

M protein encoded by the emm gene (Fischetti, 1989) has been exploited for $\mathrm{M}$ typing in epidemiological studies (Tewodros \& Kronvall, 2005). M typing has progressed from identification methods using antisera to analysis of $\mathrm{emm}$ gene sequences of the N-terminal hypervariable region using the Centers for Disease Control and

Abbreviations: AOM, acute otitis media; CDC, Centers for Disease Control and Prevention; FQ, fluoroquinolone; GAS, group A streptococcus.
Prevention (CDC) database (Beall et al., 1996). Results for GAS surveillance by Rogers et al. (2007) demonstrated that emm1 predominated in invasive infections compared with other noninvasive infections.

Macrolide and levofloxacin resistance have gradually increased in GAS isolates, although the isolates remain uniformly susceptible to $\beta$-lactam antibiotics. Clonal spread of levofloxacin-resistant GAS has been reported (Malhotra-Kumar et al., 2005), and possible implications for public health have been pointed out.

Recently, penicillin-intermediately resistant Streptococcus agalactiae that has amino acid substitutions in penicillinbinding protein $2 \mathrm{X}$ has appeared in Japan (Kimura et al., 2008). Therefore, attention to annual trends of $\beta$-lactam susceptibility of GAS is also necessary.

In this study, we aimed to clarify (i) emm-type distributions in invasive strains compared with noninvasive isolates; (ii) susceptibility of these strains to $11 \beta$-lactam antibiotics, four macrolides, clindamycin and levofloxacin; 
and (iii) resistance genes for macrolides, lincosamides and streptogramin B $\left(\mathrm{MLS}_{\mathrm{B}}\right)$, and levofloxacin.

\section{METHODS}

Strains. GAS isolates from patients with GAS infection were collected from clinical laboratories belonging to 45 general hospitals. A total of 482 isolates were sent to our laboratory (Kitasato Institute for Life Sciences, Kitasato University) between October 2003 and September 2006. After reidentification following the Manual of Clinical Microbiology (Ruoff et al., 2003), strains were stored at $-80{ }^{\circ} \mathrm{C}$ in $10 \%$ skim milk (Becton Dickinson) until subsequent testing.

For the purposes of this study, isolates were divided into four groups according to the modified classification of Rogers et al. (2007). The invasive infection group (total $n=74)$ included septicaemia $(n=34)$, cellulitis $(n=13)$, septic arthritis $(n=8)$, meningitis $(n=4)$, necrotizing fasciitis $(n=5)$, toxic-shock-like syndrome $(n=4)$, pneumonia $(n=3)$, mastitis $(n=2)$ and pleurisy $(n=1)$ isolates. In this group, isolates defined tentatively as GAS were obtained from blood, joint fluid or pleural fluid. The other GAS infections were classified in abscess $(n=53)$, pharyngotonsillitis $(n=332)$ and acute otitis media $(\mathrm{AOM})(n=23)$ groups.

Characterization of the resistance mechanism. PCRs for detection of the $\operatorname{erm}(\mathrm{A}), \operatorname{erm}(\mathrm{B})$ and $m e f(\mathrm{~A})$ genes mediating macrolide resistance were performed as described previously (Sunaoshi et al., 2004).

Strains possessing the erm(A) gene express an inducible macrolide, lincosamide and streptogramin $\mathrm{B}$ resistance $\left(\mathrm{iMLS}_{\mathrm{B}}\right)$ phenotype (Seppälä et al., 1998) and strains possessing the $\operatorname{erm}(\mathrm{B})$ gene express a constitutive $\mathrm{MLS}_{\mathrm{B}}\left(\mathrm{cMLS}_{\mathrm{B}}\right)$ phenotype. The $\mathrm{iMLS}_{\mathrm{B}}$ phenotype strains by the target site modifications due to methylase activity show high resistance to 14- and 15-membered ring macrolides but are susceptible or intermediately resistant to clindamycin without induction (Giovanetti et al., 1999). The $\mathrm{CMLS}_{\mathrm{B}}$ phenotype strains by methylase activity show high resistance to all macrolides and clindamycin without induction. By contrast, strains possessing the mef(A) gene express a 14- and 15-membered ring macrolide resistance (M) phenotype by an active drug efflux pump (Giovanetti et al., 1999; Roberts et al., 1999).

For the gyrA, gyrB, parC and parE genes involved in fluoroquinolone (FQ) resistance, PCR was carried out for 40 cycles under the conditions of $94{ }^{\circ} \mathrm{C}$ for $30 \mathrm{~s}, 52{ }^{\circ} \mathrm{C}$ for $30 \mathrm{~s}$ and $72{ }^{\circ} \mathrm{C}$ for $60 \mathrm{~s}$. Analytical primer sets for FQ resistance, shown in Table 1, were designed to detect these genes in GAS strains (GenBank accession nos NC_002737, NC_008023, NC_008024). The PCR product was then purified using the QIAquick PCR Purification kit (Qiagen). Sequencing was performed using the BigDye Terminator Cycle Sequencing kit version 3.1 (Applied Biosystems), with assessment of results using the Applied Biosystems 3130 Genetic Analyzer.

emm gene typing. Typing of the emm gene was performed as follows.

Extraction of template DNA was done using the established procedure (Ubukata et al., 1996). In brief, one colony of GAS growing on a sheep blood agar culture plate was picked and suspended in lysis solution, which comprised $0.1 \mathrm{M} \mathrm{Tris} / \mathrm{HCl}$ $(\mathrm{pH} 8.0), 4 \mu \mathrm{g}$ proteinase $\mathrm{K}, 0.225 \%$ Tween 20 and $0.225 \%$ Nonidet 40. This was incubated at $60{ }^{\circ} \mathrm{C}$ for $20 \mathrm{~min}$ and then at $90{ }^{\circ} \mathrm{C}$ for $10 \mathrm{~min}$.

The PCR for emm genotyping was carried out according to minor modifications of the method described previously by Beall et al.
Table 1. Primers for PCR and sequencing for quinolone resistance

\begin{tabular}{|c|c|c|}
\hline Primer name & Primer sequence $\left(5^{\prime} \rightarrow 3^{\prime}\right)$ & $\begin{array}{c}\text { Amplicon size } \\
\text { (bp) }\end{array}$ \\
\hline \multicolumn{3}{|l|}{ gyrA } \\
\hline gyrA-s & TTTGCCAGATGTGCGTGATG & 446 \\
\hline gyrA-r & TGTTAGTTGCCATCCCAACG & \\
\hline \multicolumn{3}{|l|}{ gyrB } \\
\hline gyrB-s & ATTGGGCAACTCAGAAGTGG & 504 \\
\hline gyrB-r & GTTCTAATATGAGCGCCATCC & \\
\hline \multicolumn{3}{|l|}{ parC } \\
\hline parC-s & ATTATGGGAGAACGCTTCGG & 442 \\
\hline parC-r & AAGCTGCTGGTAAAACGGTG & \\
\hline \multicolumn{3}{|l|}{ parE } \\
\hline parE-s & CTCATCTAGTTCGCAAAGCC & 781 \\
\hline parE-r & TTATCCTCGATCCACTGACG & \\
\hline
\end{tabular}

(1996). The resulting PCR fragments were purified and sequenced in the same way as described above.

The first 240 bases of the $5^{\prime}$ end of the emm gene sequences were compared with those in the CDC S. pyogenes emm sequence database (http://www.cdc.gov/ncidod/biotech/strep/strepblast.htm). An emm type showing over $98 \%$ homology with a CDC reference strain was identified as that particular emm type.

Antimicrobial susceptibility tests. Susceptibility testing of GAS strains was carried out by the microdilution method using cationadjusted Mueller-Hinton broth (Becton Dickinson) supplemented with $5 \%$ lysed horse blood according to the guidelines of the Clinical and Laboratory Standards Institute (CLSI, 2007) and with a final inoculum of $5 \times 10^{5}$ c.f.u. $\mathrm{ml}^{-1}$. Oral antimicrobial agents employed in this study were ampicillin, amoxicillin, cefdinir, cefpodoxime, cefditoren, cefcapen, faropenem, tebipenem, clarithromycin, azithromycin, josamycin, clindamycin, telithromycin and levofloxacin. Parenteral agents were cefotaxime, panipenem and meropenem. These antimicrobial agents were obtained from the respective pharmaceutical manufacturers.

Statistical analysis. Statistical analysis was performed using Microsoft Excel Statistics 2006 for Windows (Social Survey Research Information). The chi-square test was used to assess significance of differences involving categorical variables.

\section{RESULTS AND DISCUSSION}

\section{Age distribution of the patients with GAS infection}

Fig. 1 shows the patient age distribution in the four groups of GAS infection cases described in Methods.

Invasive cases $(n=74)$ occurred mostly in patients over 20 years old $(85.1 \%)$ as opposed to in children, especially in older adults between the ages of 50 and 70 years old. Important underlying conditions such as diabetes mellitus, liver dysfunction, renal dysfunction and medical treatment for cancer were noted in $56.4 \%$ of those adults. Decreased immunity in these cases may have contributed synergisti- 


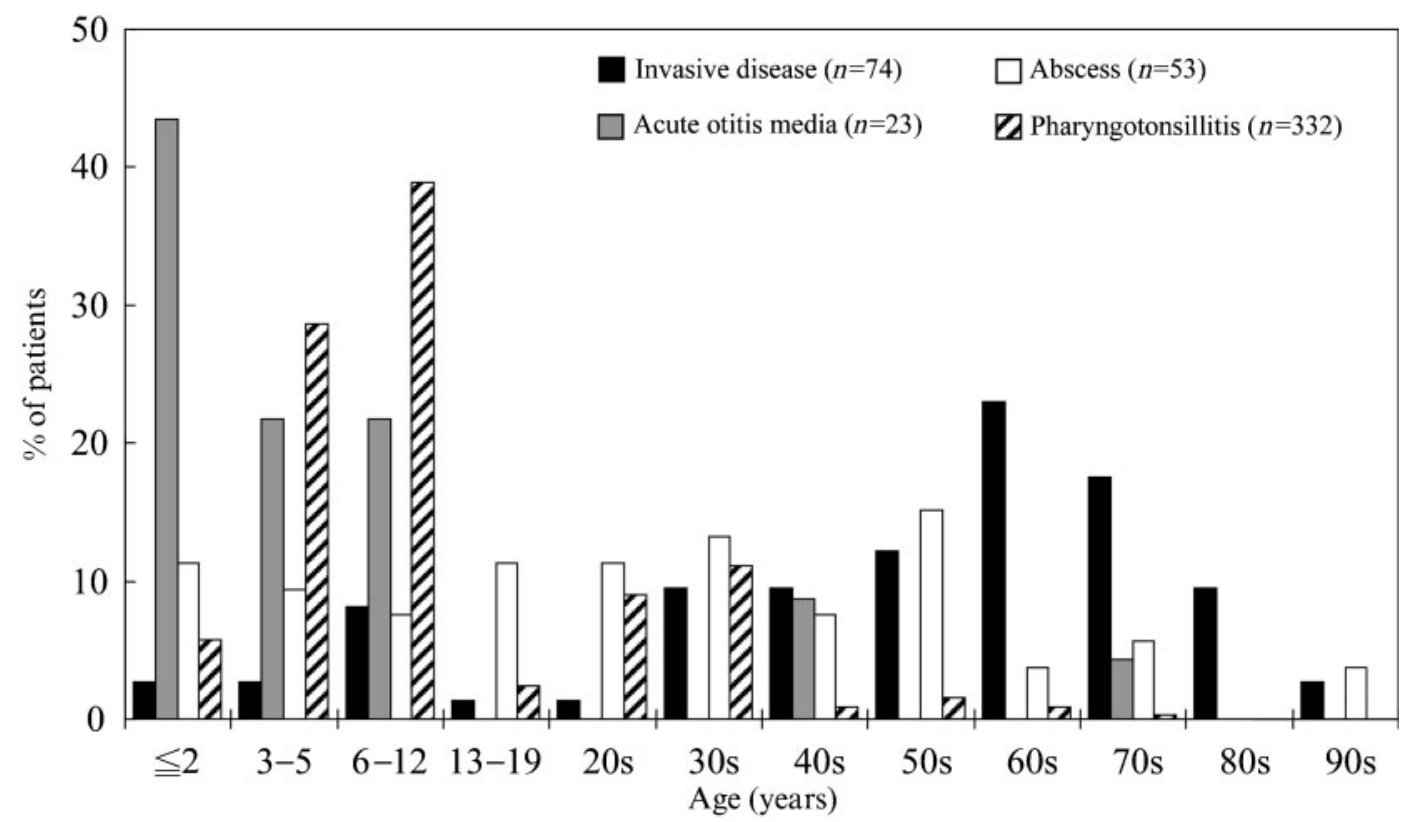

Fig. 1. Age distribution of patients with Streptococcus pyogenes infection. Patients were classified into four groups. Black columns indicate patients with invasive infections; finely stippled columns, those with AOM; open columns, those with abscess; diagonally hatched columns, those with pharyngotonsillitis.

cally with GAS virulence factors to an increased incidence of invasive infections.

Both AOM cases $(n=23)$ and pharyngotonsillitis cases $(n=332)$ mainly involved children between 3 and 12 years old, which accounted for $43.5 \%$ and $67.4 \%$, respectively, although $20.1 \%$ of the pharyngotonsillitis patients were 20-30 years old.

Abscess cases $(n=53)$, in which GAS was isolated from either draining or nondraining localized abscesses, were distributed across all age brackets.

Statistically significant differences in age distribution were recognized between the invasive group and each of the other three groups (invasive vs AOM, $P<0.0001$; invasive vs abscess, $P=0.0003$; invasive vs pharyngotonsillitis, $P$ $<0.0001)$.

\section{Typing for emm}

Results of emm typing of GAS isolates in four groups, that is, invasive (A), AOM (B), abscess (C) and pharyngotonsillitis (D) groups, are listed in Table 2. Although GAS isolates included a variety of emm types, the predominant $\mathrm{emm}$ types in each group differed. In the invasive group, emm 1 was prominent, accounting for $39.2 \%$ of cases, while other emm types generally accounted for $10 \%$ of cases or fewer. Similarly, emm1 was most frequent in the AOM group $(43.5 \%)$, followed by emm $12(30.4 \%)$.

In the abscess group, emm 28 and types described as 'other' in Table 2 accounted for $22.6 \%$ and $26.4 \%$ of cases each, while emm1 was responsible for only $3.8 \%$. In the pharyngotonsillitis group, emm 4 and emm12 accounted for $23.5 \%$ of cases each, in contrast to $10.2 \%$ for emm 1 .

A significant difference in prevalence of emm types was noted between the invasive group and each of the other three groups (invasive vs AOM, $P=0.0895$; invasive vs abscess, $P=0.0013$; invasive vs pharyngotonsillitis, $P$ $<0.0001)$.

\section{Susceptibilities to $\beta$-lactam agents}

All $\beta$-lactam agents showed sharp distributions for all GAS, indicating that none of these agents showed decreased efficacy against GAS.

In data not shown here, $\mathrm{MIC}_{90}$ values for GAS were excellent in the following order: tebipenem $(0.002 \mu \mathrm{g}$ $\left.\mathrm{ml}^{-1}\right)>$ cefditoren $=$ cefcapen $\left(0.008 \mu \mathrm{g} \mathrm{ml}^{-1}\right)>$ amoxicillin $=$ cefdinir $=$ cefpodoxime $\left(0.016 \mu \mathrm{g} \mathrm{ml}^{-1}\right)>$ ampicillin $=$ faropenem $\left(0.031 \mu \mathrm{g} \mathrm{ml}^{-1}\right)$ for oral $\beta$-lactams; and panipenem $=$ meropenem $\left(0.008 \mu \mathrm{g} \mathrm{ml}^{-1}\right)>$ cefotaxime $\left(0.016 \mu \mathrm{g} \mathrm{ml}^{-1}\right)$ for parenteral $\beta$-lactams. All isolates remained uniformly susceptible to $\beta$-lactam antibiotics.

\section{Susceptibilities to macrolides and resistance genes}

Table 3 shows the MIC ranges, $\mathrm{MIC}_{50}$ and $\mathrm{MIC}_{90}$ of clarithromycin, azithromycin, josamycin, telithromycin and clindamycin for GAS according to the macrolideresistance genes identified. Of all 482 isolates, strains 
Table 2. Relationship between streptococcal diseases and emm types of isolates

Results of chi-square tests were as follows. A: B, $P=0.0895$; A:C, $P=0.0013, \mathrm{~A}: \mathrm{D}, P<0.0001$. Values in parentheses are percentages for each classified group. AOM, acute otitis media.

\begin{tabular}{|c|c|c|c|c|c|}
\hline \multirow[t]{2}{*}{ emm type } & \multicolumn{4}{|c|}{ Groups classified as: } & \multirow[t]{2}{*}{ No. of isolates } \\
\hline & Invasive (A) & AOM (B) & Abscess (C) & Pharyngotonsillitis (D) & \\
\hline 1 & $29(39.2)$ & $10(43.5)$ & $2(3.8)$ & $34(10.2)$ & 75 \\
\hline 3 & $2(2.7)$ & & $2(3.8)$ & $13(3.9)$ & 17 \\
\hline 4 & $2(2.7)$ & $2(8.7)$ & $1(1.9)$ & $78(23.5)$ & 83 \\
\hline 6 & & & $3(5.7)$ & $15(4.5)$ & 18 \\
\hline 11 & $4(5.4)$ & & $2(3.8)$ & $20(6.0)$ & 26 \\
\hline 12 & $5(6.7)$ & $7(30.4)$ & $4(7.5)$ & $78(23.5)$ & 94 \\
\hline 28 & $5(6.7)$ & $1(4.3)$ & $12(22.6)$ & $33(9.9)$ & 51 \\
\hline 49 & $8(10.8)$ & & $4(7.5)$ & $1(0.3)$ & 13 \\
\hline 58 & $4(5.4)$ & & $3(5.7)$ & $7(2.1)$ & 14 \\
\hline 75 & $3(4.1)$ & $2(8.7)$ & $1(1.9)$ & $9(2.7)$ & 15 \\
\hline 87 & $2(2.7)$ & & $2(3.8)$ & $2(0.6)$ & 6 \\
\hline 89 & $1(1.4)$ & $1(4.3)$ & $3(5.7)$ & $12(3.6)$ & 17 \\
\hline Other & $9(12.2)$ & & $14(26.4)$ & $30(9.0)$ & 53 \\
\hline Total & 74 & 23 & 53 & 332 & 482 \\
\hline
\end{tabular}

possessing $\mathrm{erm}(\mathrm{A}), \mathrm{erm}(\mathrm{B})$ and $m e f(\mathrm{~A})$ represented $2.5 \%$ $(n=12), 6.2 \%(n=30)$ and $7.5 \%(n=36)$, respectively.

Table 3. MIC distributions and resistance genes identified by PCR in Streptococcus pyogenes strains

\begin{tabular}{|c|c|c|c|}
\hline Antimicrobial agent & 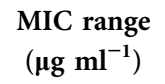 & $\mathrm{MIC}_{50}$ & $\mathrm{MIC}_{90}$ \\
\hline \multicolumn{4}{|l|}{ Clarithromycin } \\
\hline Resistance gene $(-)$ & $0.031-2$ & 0.063 & 0.125 \\
\hline $\operatorname{erm}(\mathrm{A})$ & $2-16$ & 4 & 16 \\
\hline $\operatorname{erm}(\mathrm{B})$ & $>64$ & $>64$ & $>64$ \\
\hline$m e f(\mathrm{~A})$ & $2-16$ & 8 & 16 \\
\hline \multicolumn{4}{|l|}{ Azithromycin } \\
\hline Resistance gene $(-)$ & $0.125-4$ & 0.25 & 1 \\
\hline $\operatorname{erm}(\mathrm{A})$ & $32->64$ & $>64$ & $>64$ \\
\hline $\operatorname{erm}(\mathrm{B})$ & $>64$ & $>64$ & $>64$ \\
\hline$m e f(\mathrm{~A})$ & $4-32$ & 8 & 32 \\
\hline \multicolumn{4}{|l|}{ Josamycin } \\
\hline Resistance gene $(-)$ & $0.125-2$ & 0.5 & 0.5 \\
\hline $\operatorname{erm}(\mathrm{A})$ & $0.5-8$ & 4 & 8 \\
\hline $\operatorname{erm}(\mathrm{B})$ & $>64$ & $>64$ & $>64$ \\
\hline$m e f(\mathrm{~A})$ & $0.125-0.5$ & 0.25 & 0.5 \\
\hline \multicolumn{4}{|l|}{ Telithromycin } \\
\hline Resistance gene $(-)$ & $0.008-0.25$ & 0.031 & 0.063 \\
\hline $\operatorname{erm}(\mathrm{A})$ & $0.063-0.125$ & 0.063 & 0.063 \\
\hline $\operatorname{erm}(\mathrm{B})$ & $2->64$ & $>64$ & $>64$ \\
\hline$m e f(\mathrm{~A})$ & $0.25-1$ & 0.5 & 1 \\
\hline \multicolumn{4}{|l|}{ Clindamycin } \\
\hline Resistance gene $(-)$ & $0.031-0.25$ & 0.125 & 0.25 \\
\hline $\operatorname{erm}(\mathrm{A})$ & $0.25->64$ & 0.5 & 8 \\
\hline $\operatorname{erm}(\mathrm{B})$ & $>64$ & $>64$ & $>64$ \\
\hline$m e f(\mathrm{~A})$ & $0.031-0.25$ & 0.063 & 0.25 \\
\hline
\end{tabular}

Except for telithromycin, the erm(A) gene related to iMLS phenotype decreased the susceptibilities of GAS to macrolides and clindamycin 8-128-fold compared with susceptible strains. GAS with the $\operatorname{erm}(\mathrm{B})$ gene related to $\mathrm{CMLS}_{\mathrm{B}}$ phenotype showed high resistance to macrolides, telithromycin and clindamycin ( $\mathrm{MIC} \geqslant 64 \mu \mathrm{g} \mathrm{ml}^{-1}$ ). GAS strains with the mef(A) gene, which mediate an $M$ phenotype, showed slight decreases in susceptibility, from 4- to 8-fold, to clarithromycin, azithromycin and telithromycin, but maintained undiminished susceptibility to josamycin and clindamycin.

In Japan, the prevalence of macrolide resistance in GAS has remained at 5-7 \% for a long time. In the survey in 2003, of the total of 533 GAS strains collected from all over the country, $0.5 \%$ had the erm(A) gene, $3.2 \%$ the $\operatorname{erm}(\mathrm{B})$ gene and $4.9 \%$ the mef(A) gene (Sunaoshi et al., 2004). Macrolide resistance appears to increase gradually.

Table 4 shows the correlation between the emm type and macrolide-resistance genes. Of the GAS strains typed to emm $1,21.3 \%$ had the mef(A) gene, which was prevalent in invasive infections, in contrast to the emm status of strains with $\operatorname{erm}(\mathrm{A})$ and $\operatorname{erm}(\mathrm{B})$ genes.

High clindamycin resistance mediated by the erm(B) gene was detected at $2.7 \%$ in invasive strains.

\section{Susceptibility to levofloxacin and mutations of the target gene}

Table 4 also shows the emm type distribution of GAS strains $(n=71)$ which were intermediately resistant to FQ. Although no strain was identified as showing high resistance, strains with an MIC of $2-4 \mu \mathrm{g} \mathrm{ml} \mathrm{m}^{-1}$ accounted for $17.4 \%(n=84)$ of all isolates. Eighty-five per cent of 
Table 4. Correlation of emm type and resistance genes of Streptococcus pyogenes strains

Values in parentheses are percentages for each emm type.

\begin{tabular}{|c|c|c|c|c|c|}
\hline \multirow[t]{2}{*}{ emm type } & \multirow[t]{2}{*}{ No. of isolates } & \multicolumn{3}{|c|}{ Macrolide resistance } & \multirow[t]{2}{*}{ Fluoroquinolone resistance parC mutant ${ }^{\star}$} \\
\hline & & $\operatorname{erm}(\mathrm{A})$ & $\operatorname{erm}(\mathrm{B})$ & $m e f(\mathrm{~A})$ & \\
\hline 3 & 17 & & & & $1(5.9)$ \\
\hline 4 & 83 & & & $6(7.2)$ & $1(1.2)$ \\
\hline 6 & 18 & & & & $17(94.4)$ \\
\hline 28 & 51 & $3(5.9)$ & $14(27.4)$ & & $2(3.9)$ \\
\hline 49 & 13 & & & & $4(30.8)$ \\
\hline 58 & 14 & $6(42.8)$ & & & $3(21.4)$ \\
\hline 75 & 15 & & & $7(46.7)$ & $1(6.7)$ \\
\hline 87 & 6 & & & & $5(83.3)$ \\
\hline
\end{tabular}

${ }^{\star}$ Ser-79 and Asp-83 in ParC encoded by parC changed to Phe, Ala, or Tyr and Asn.

strains with an MIC of at least $\geqslant 2 \mu \mathrm{g} \mathrm{ml}^{-1}(n=71)$ had an amino acid substitution at the Ser-79 or Asp-83 position in quinolone resistance-determining regions (QRDRs) encoded by parC. Ser-79 was changed to Phe- 79 , Ala-79 or Tyr-79 in $93.0 \%(n=66)$, while Asp-83 was changed to Asn83 in $7.0 \%(n=5)$. There was no amino acid substitution affecting FQ resistance in $g y r A, g y r B$ and parE genes.

Genotypic levofloxacin-intermediately resistant GAS strains belonged to $16 \mathrm{emm}$ types, although emm6 and emm 11 were prominent at $94.4 \%$ and $76.9 \%$, respectively, as described previously (Orscheln et al., 2005). This finding suggests that genotypically FQ-intermediately resistant GAS is selected under the pressure of exposure to FQs including levofloxacin. In vitro experiments have indicated that all emm types seem equally prone to induction of FQ resistance (Billal et al., 2007).

Recently, we isolated a GAS strain, showing high resistance to $\mathrm{FQ}$, from an adult patient (29 years old) with pharyngotonsillitis in September 2007. The strain possessed amino acid substitutions in QRDRs of both gyrA and parC, which had already been reported in several countries (Malhotra-Kumar et al., 2005; Reinert et al., 2004; Richter et al., 2003; Rivera et al., 2005; Yan et al., 2000).

According to the market research for oral antibiotics (Fujita et al., 2007) in Japan, oral FQs, including four respiratory FQs, have been prescribed for adult outpatients aged $\geqslant 15$ years at the highest rate of $50 \%$, followed by oral cephalosporin antibiotics at the rate of $43 \%$, which is higher than that for penicillins. The status of the current usage of FQ causes concern that the incidence of GAS and Streptococcus pneumoniae strains possessing high FQ resistance might increase in the near future.
On the other hand, macrolides to which 14-membered ring macrolides and azalides belong have been widely prescribed to reduce inflammation in patients with diffuse panbronchiolitis, chronic bronchitis and chronic sinusitis at low doses for long periods, in addition to being used in the treatment of community-acquired respiratory tract infections. Such long-term non-chemotherapeutical usage of macrolides may result in a decrease in usefulness of macrolides for infectious disease and be related to selection and spread of macrolide resistance in S. pneumoniae and $S$. pyogenes.

In conclusion, continuous molecular epidemiological surveillance of GAS is necessary to ensure proper use of antimicrobial agents.

\section{ACKNOWLEDGEMENTS}

This work was supported by a grant from the Japanese Ministry of Health, Labour, and Welfare (Research Project for Emerging and Reemerging Infectious Diseases, H19-002).

\section{REFERENCES}

Beall, B., Facklam, R. \& Thompson, T. (1996). Sequencing emmspecific PCR products for routine and accurate typing of group A streptococci. J Clin Microbiol 34, 953-958.

Billal, D. S., Fedorko, D. P., Yan, S. S., Hotomi, M., Fujihara, K., Nelson, N. \& Yamanaka, N. (2007). In vitro induction and selection of fluoroquinolone-resistant mutants of Streptococcus pyogenes strains with multiple emm types. J Antimicrob Chemother 59, 28-34.

CLSI (2007). Performance Standards for Antimicrobial Susceptibility Testing, 17th Informational Supplement, M100-S17. Wayne, PA: Clinical and Laboratory Standards Institute. 
Cunningham, M. W. (2000). Pathogenesis of group A streptococcal infections. Clin Microbiol Rev 13, 470-511.

Fischetti, V. A. (1989). Streptococcal M protein: molecular design and biological behavior. Clin Microbiol Rev 2, 285-314.

Fujita, T., Kawaguchi, T. \& Fujii, Y. (2007). The closeout report: database construction of oral antimicrobial agents using the results from drug use investigations. Jpn J Antibiot 60, 265-307 (in Japanese).

Giovanetti, E., Montanari, M. P., Mingoia, M. \& Varaldo, P. E. (1999). Phenotypes and genotypes of erythromycin-resistant Streptococcus pyogenes strains in Italy and heterogeneity of inducibly resistant strains. Antimicrob Agents Chemother 43, 1935-1940.

Kimura, K., Suzuki, S., Wachino, J. I., Kurokawa, H., Yamane, K., Shibata, N., Nagano, N., Kato, H., Shibayama, K. \& Arakawa, Y. (2008). First molecular characterization of group B streptococci with reduced penicillin susceptibility. Antimicrob Agents Chemother 52, 2890-2897.

Malhotra-Kumar, S., Lammens, C., Chapelle, S., Mallentjer, C., Weyler, J. \& Goossens, H. (2005). Clonal spread of fluoroquinolone non-susceptible Streptococcus pyogenes. J Antimicrob Chemother 55, 320-325.

Orscheln, R. C., Johnson, D. R., Olson, S. M., Presti, R. M., Martin, J. M., Kaplan, E. L. \& Storch, G. A. (2005). Intrinsic reduced susceptibility of serotype 6 Streptococcus pyogenes to fluoroquinolone antibiotics. J Infect Dis 191, 1272-1279.

Reinert, R. R., Lutticken, R. \& Al-Lahham, A. (2004). High-level fluoroquinolone resistance in a clinical Streptococcus pyogenes isolate in Germany. Clin Microbiol Infect 10, 659-662.

Richter, S. S., Diekema, D. J., Heilmann, K. P., Almer, L. S., Shortridge, V. D., Zeitler, R., Flamm, R. K. \& Doern, G. V. (2003). Fluoroquinolone resistance in Streptococcus pyogenes. Clin Infect Dis 36, 380-383.

Rivera, A., Rebollo, M., Sanchez, F., Navarro, F., Miro, E., Mirelis, B. \& Coll, P. (2005). Characterisation of fluoroquinolone-resistant clinical isolates of Streptococcus pyogenes in Barcelona, Spain. Clin Microbiol Infect 11, 759-761.
Roberts, M. C., Sutcliffe, J., Courvalin, P., Jensen, L. B., Rood, J. \& Seppälä, H. (1999). Nomenclature for macrolide and macrolidelincosamide-streptogramin B resistance determinants. Antimicrob Agents Chemother 43, 2823-2830.

Rogers, S., Commons, R., Danchin, M. H., Selvaraj, G., Kelpie, L., Curtis, N., Robins-Browne, R. \& Carapetis, J. R. (2007). Strain prevalence, rather than innate virulence potential, is the major factor responsible for an increase in serious group A streptococcus infections. J Infect Dis 195, 1625-1633.

Ruoff, K. L., Whiley, R. A. \& Beighton, D. (2003). Streptococcus. In Manual of Clinical Microbiology, pp. 405-421. Edited by P. R. Murray, E. J. Baron, J. H. Jorgensen, M. A. Pfaller \& R. H. Yolken. Washington, DC: American Society for Microbiology.

Seppälä, H., Skurnik, M., Soini, H., Roberts, M. C. \& Huovinen, P. (1998). A novel erythromycin resistance methylase gene (ermTR) in Streptococcus pyogenes. Antimicrob Agents Chemother 42, 257-262.

Stevens, D. L., Tanner, M. H., Winship, J., Swarts, R., Ries, K. M., Schlievert, P. M. \& Kaplan, E. (1989). Severe group A streptococcal infections associated with a toxic shock-like syndrome and scarlet fever toxin A. N Engl J Med 321, 1-7.

Sunaoshi, K., Nakayama, E., Kobayashi, R., Suzuki, E., Tajima, T. \& Ubukata, K. (2004). Antibiotic susceptibility and T type identification of Streptococcus pyogenes isolated from pediatric outpatients with pharingotonsillitis. Jpn J Chemother 52, 401-407.

Tewodros, W. \& Kronvall, G. (2005). M protein gene (emm type) analysis of group A beta-hemolytic streptococci from Ethiopia reveals unique patterns. J Clin Microbiol 43, 4369-4376.

Ubukata, K., Asahi, Y., Yamane, A. \& Konno, M. (1996). Combinational detection of autolysin and penicillin-binding protein 2B genes of Streptococcus pneumoniae by PCR. J Clin Microbiol 34, 592-596.

Yan, S. S., Fox, M. L., Holland, S. M., Stock, F., Gill, V. J. \& Fedorko, D. P. (2000). Resistance to multiple fluoroquinolones in a clinical isolate of Streptococcus pyogenes: identification of gyrA and parC and specification of point mutations associated with resistance. Antimicrob Agents Chemother 44, 3196-3198. 